Draft VERsion November 5, 2020

Typeset using IATEX RNAAS style in AASTeX63

\title{
The outburst of Centaur 29P/Schwassmann-Wachmann on February 4th 2020
}

\author{
Abbie Donaldson ${ }^{1}$ and Aleks Scholz ${ }^{1}$ \\ ${ }^{1}$ SUPA, School of Physics $\&$ Astronomy, University of St Andrews, North Haugh, St Andrews, KY16 9SS, United Kingdom
}

\begin{abstract}
We observed the February 4th 2020 outburst of the Centaur 29P/Schwassmann-Wachmann, using the James Gregory Telescope in St Andrews/UK. The brightness of the comet increased by $\sim 3 \mathrm{mag}$ in VRI within 1-2 d, followed by a more gradual incline. This was one of the largest documented outbursts of this object over the past few years, but is consistent with previously observed events.
\end{abstract}

Comet 29P/Schwassmann-Wachmann (29P in the following) is an enigmatic member of the Centaurs, unique in its persistent and high level of activity. Frequently the comet shows sudden dusty outbursts when the optical brightness increases by 1-4 mag (Trigo-Rodríguez et al. 2010). In recent years, about 6-9 outbursts occur per annum, lasting from a few hours to several days (Miles 2018). The origin of the outbursts is still poorly understood. With a fairly circular orbit and a perihelion distance of 5.7 AU, the object experiences similar levels of insolation at all times. This implies that its activity is not thermally driven. The outbursts are also not always correlated with increased CO production (Wierzchos \& Womack 2020). Continuous monitoring is needed to be able to trigger detailed follow-up during outbursts.

In early October 2019, signs of a possible fragmentation event were reported for 29P (Kelley et al. 2019), followed by a low-level outburst (Ye et al. 2019). Motivated by this event, we decided to monitor the comet over the winter 2019/20, using the James Gregory Telescope (JGT) in St Andrews/UK. The JGT is a vintage 37" Schmidt-Cassegrain optics (Fraser 1977), operated currently at 33" aperture. We used a QSI512 CCD camera with $9 \mu \mathrm{m}$ pixel size, corresponding to $0.73 " /$ pixel, with a total field of view of $24^{\prime} \times 16^{\prime}$. The camera has an built-in filter wheel with standard BVRI filters. The telescope is operated by volunteer student observers. St Andrews is at sea level; the resulting poor seeing of several arcsec prevents detailed study of the comet's coma. We are therefore only reporting integrated photometry.

In each suitable night, we obtained 1-3 sequences of $10 \times 60 \mathrm{~s}$ exposures in each of the three filters $\mathrm{V}, \mathrm{R}$,

Corresponding author: Aleks Scholz

as110@st-andrews.ac.uk and I. We observed the comet on 6 nights, from November 12th 2019 to February 6th 2020. In November (2 epochs), December (1 epoch), and January (1 epoch), the comet's brightness was comparable to typical levels of quiescence; we report here only the values obtained on December 17th 2019 (17.8). In contrast, on February 4th (4.7) and February 6th (6.8) the object was significantly brighter. From mid December to early February, the geocentric distance of the comet increased from 5.4 to $6.2 \mathrm{AU}$, while the heliocentric distance of $5.8 \mathrm{AU}$ does not change notably.

We performed aperture photometry on the comet and comparison stars on the stacked $10 \times 60$ s sets, and averaged over multiple sets per night, if available. The stacking was done twice, once tracking the moving object and once for sidereal tracking. We used an aperture radius twice the FWHM (corresponding to 7.3 to 8.0 ") as well as an aperture corresponding to $15000 \mathrm{~km}$ radius at the comet's distance (corresponding to 3.3 to 3.8 "). The latter aperture only encompasses the core of the point spread function and the fluxes become seeing dependent. The photometry was shifted to Johnson V, Sloan $\mathrm{r}$ and i, using calibrated magnitudes from APASS as reported in UCAC4 (Zacharias et al. 2013). To account for the colour shift between Johnson and Sloan systems, we used transformations published by Lupton ${ }^{1}$. The typical photometry uncertainty is $0.1 \mathrm{mag}$, combining measurement and calibration errors.

The lightcurve with $2 \times \mathrm{FWHM}$ aperture is shown in Fig. 1. In $\mathrm{V} / \mathrm{R} / \mathrm{I}$ we measure $17.0 / 16.5 / 15.9$, $13.8 / 13.3 / 12.8,14.4 / 13.8 / 13.3$ for the three epochs with the described setup. The comet increased in brightness by 3.1-3.2 mag during the outburst, and faded by 0.5 $0.6 \mathrm{mag}$ a couple of days later. There is no discernible colour change in our photometry. Converting these val-

${ }^{1}$ www.sdss.org/dr16/algorithms/sdssubvritransform/ 


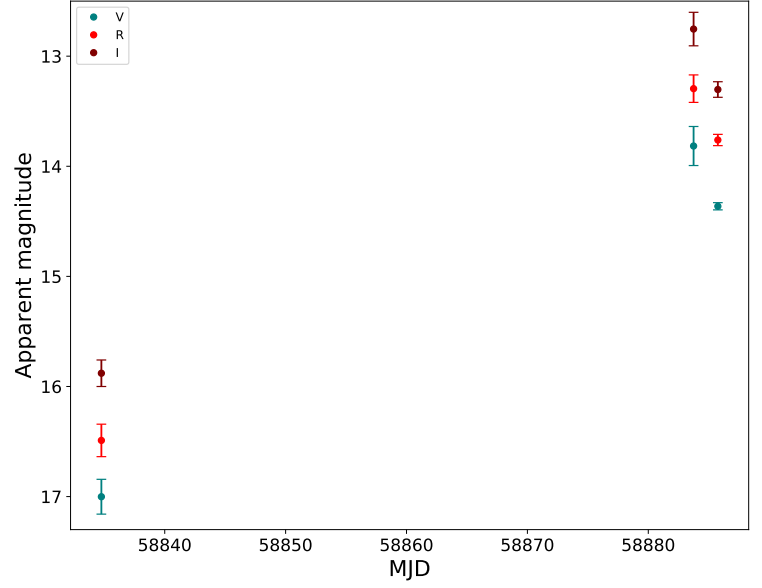

Figure 1. Multi-band lightcurve from three epochs of JGT photometry for 29P/Schwassmann-Wachmann, using an aperture of twice the FWHM.

ues to absolute magnitudes in $\mathrm{V} / \mathrm{R} / \mathrm{I}$ gives $8.8 / 8.3 / 7.7$, $5.3 / 4.8 / 4.2$, and 5.8/5.2/4.8 for the three epochs, an increase of 3.5 mag during the outburst. For this conversion, we used tabulated ${ }^{2}$ values for the phase function to normalise to phase angle $0^{\circ}$ (Schleicher et al. 1998). For the smaller, $15000 \mathrm{~km}$ aperture, the apparent magnitude also increased by about 3 mag during the outburst. From these values, we calculated the Af $\rho$ parameter, often used to quantify dust production (Trigo-Rodríguez et al. 2010). In the R-band, Af $\rho$ rises from $1760 \pm 200 \mathrm{~cm}$ in quiescence to $32590 \pm 3740 \mathrm{~cm}$ in outburst.

The event was also reported by various other observers $^{3}$, although observations were generally hampered by the bright moon and the short visibility window for the target. Magnitudes given by Richard Miles are consistent with quiescence up until Feb 2.1. Quanzhi
Ye finds a g-band magnitude of 14.35 on Feb 2.56, while Brian Skiff reports an r-band magnitude of 13.42 on Feb 3.1 (comparable with our own value on Feb 4.7). A visual magnitude of 12.0 was provided by Bob King for Feb 4.1. These measurements are not necessarily comparable to our values, due to possible differences in aperture or filter curve. They do indicate that the outburst started during Feb 2nd and reached maximum brightness within a day or two. It is plausible that the peak brightness occurred between Feb 3.1 and our measurement on Feb 4.7. Our photometry on Feb 6.8 indicates a much slower decline back to quiescent levels.

Reaching an absolute magnitude of about 5 in the rband near peak, this was one of the largest outbursts of $29 \mathrm{P}$ documented within the last few years. As far as is known, the characteristics are comparable to previously reported large outbursts, with a sudden increase and a gradual decline, with only small changes in colour (undetected in our photometry). The increase in Af $\rho$ is also consistent with previously observed events (TrigoRodríguez et al. 2010). The February 4th 2020 outburst appears to demonstrate that the overall activity of 29P/Schwassmann-Wachmann continues after the supposed fragmentation event in October 2019.

\section{ACKNOWLEDGMENTS}

We thank the JGT observing team in winter 2019/20, in particular Izzy Donaldson, Glen Hunter, and Fergus and Callum Donnan. We also acknowledge technical support from Sam Pearson, Roger Stapleton, and Stephen King. This work has benefitted from instructive discussions with Rosita Kokotanekova and Colin Snodgrass. This research made use of the AAVSO Photometric All-Sky Survey (APASS), funded by the Robert Martin Ayers Sciences Fund and NSF AST-1412587.

\section{REFERENCES}

Fraser, C. W. 1977, A\&AS, 29, 161

Kelley, M. S. P., Bodewits, D., Ye, Q., et al. 2019, The

Astronomer's Telegram 13164, 1

Miles, R. 2018, European Planetary Science Congress,

EPSC2018-523

Sarid, G., Volk, K., Steckloff, J. K., et al. 2019, ApJL, 883, L25

Schleicher, D. G., Millis, R. L., \& Birch, P. V. 1998, Icarus, 132,397

\footnotetext{
2 asteroid.lowell.edu/comet/dustphaseHM_table.txt

${ }^{3}$ groups.io/g/comets-ml
}

Trigo-Rodríguez, J. M., García-Hernández, D. A., Sánchez, A., et al. 2010, MNRAS, 409, 1682

Wierzchos, K., \& Womack, M. 2020, AJ, 159, 136

Ye, Q., Kelley, M. S. P., \& Bodewits, D. 2019, The

Astronomer's Telegram 13179, 1

Zacharias, N., Finch, C. T., Girard, T. M., et al. 2013, AJ, 145,44 